\title{
Finite-element formulae for calculating the sectional forces of a Bernoulli-Euler beam on continuously viscoelastic foundation subjected to concentrated moving loads
}

\author{
Ping Lou \\ School of Civil Engineering and Architecture, Railway Campus, Central South University, 22 Shao-shan-nan Road, \\ Changsha, Hunan 410075, P.R. China \\ Fax: +86 731 5571736; E-mail: pinglou@mail.csu.edu.cn or pingloucsu@126.com
}

Received 9 November 2006

Revised 2 January 2007

\begin{abstract}
In the finite element method, there are shortcomings using the conventional formulae to calculate the sectional forces, i.e., the bending moment and the shear force, at any cross-section of Bernoulli-Euler beam under dynamic loads. This paper presents some new finite-element formulae overcoming the shortcomings of the conventional ones to calculate the sectional forces at any cross-section of a Bernoulli-Euler beam on continuously viscoelastic foundation subjected to concentrated moving loads. The proposed formulae can easily degenerate into the formulae for calculating the sectional forces of a simply supported or a continuous Bernoulli-Euler beam subjected to concentrated moving loads, and into the formulae for calculating the sectional forces of a Bernoulli-Euler beam on Winkler foundation under static loads. Five numerical examples including static and dynamic analyses are chosen to illustrate the application of the proposed formulae. Numerical results show: (1) compared with the conventional formulae, the proposed formulae can improve the calculation accuracy of the sectional forces of beam; (2) one should use the proposed formulae, not the conventional formulae, to calculate the sectional forces at any cross-section in Bernoulli-Euler beam.
\end{abstract}

Keywords: Sectional forces, bending moment, shear force, finite element, Bernoulli-Euler beam, concentrated moving loads

\section{Introduction}

The dynamic analysis of a beam on elastic foundation or a simply supported beam subjected to dynamic loads is widespread in engineering. Here only some of the relevant literature is mentioned. Many researchers $[5,8,9$, $11,12,15,16,20,22]$ applied the analytical methods to investigate the dynamic problem. However, for most of the engineering problems, one must rely on numerical methods since analytical methods are usually not available. In the numerical methods, the finite element method (FEM) is powerful because it allows solution to complex problems in engineering. Some researchers $[10,13,14,18,19,23-26]$ applied FEM to study the foregoing dynamic problem. It is well known that in the design of a beam on elastic foundation or a simply supported beam subjected to moving loads, the most important considerations are the vertical deflection and the bending stress, and the latter is given by using bending moment divided by the section modulus of the beam. References $[14,18,23,25,26]$ did not care for the bending moment of beam. References $[10,13,19,24]$ reported the bending moment of beam, but they did not give the formula to calculate the bending moment of beam. 
In the dynamic analysis using FEM, if the following formulae (1)-(4) are used to calculate the sectional forces of the cross-section at any point in a Bernoulli-Euler beam element on continuously viscoelastic foundation subjected to concentrated moving loads, then, errors may appear in the numerical results because of shortcomings in using the formulae (1)-(4) to calculate the sectional forces.

$$
\begin{aligned}
& M(\xi, t)=-E I \frac{\partial^{2} y(\xi, t)}{\partial \xi^{2}}=-E I \mathbf{N}^{\prime \prime} \mathbf{q}^{e} \text { for the right-hand side of cross-section at any point } \\
& Q(\xi, t)=E I \frac{\partial^{3} y(\xi, t)}{\partial \xi^{3}}=E I \mathbf{N}^{\prime \prime \prime} \mathbf{q}^{e} \text { for the right-hand side of cross-section at any point } \\
& M(\xi, t)=E I \frac{\partial^{2} y(\xi, t)}{\partial \xi^{2}}=E I \mathbf{N}^{\prime \prime} \mathbf{q}^{e} \text { for the left-hand side of cross-section at any point } \\
& Q(\xi, t)=-E I \frac{\partial^{3} y(\xi, t)}{\partial \xi^{3}}=-E I \mathbf{N}^{\prime \prime \prime} \mathbf{q}^{e} \text { for the left-hand side of cross-section any point }
\end{aligned}
$$

where $M(\xi, t)$ and $Q(\xi, t)$ respectively denote the bending moment and the shear force of cross-section at any point and time $t$, and the positive directions of $M(\xi, t)$ and $Q(\xi, t)$ for the right-hand side of cross-section and for the left-hand side of cross-section at any point are shown in Fig. 1(a) and (b), respectively; $\xi$ denotes local coordinate measured from the left node of the beam element; $E I$ denotes the constant bending stiffness of the beam; $\mathbf{N}$ denotes the shape matrix of the beam element; $\mathbf{q}^{e}$ denotes the nodal displacement vector of the beam element; the superscript prime denotes differentiation with respect to $\xi$; and $y(\xi)$ denotes the vertical deflection at any point in the beam element. $y(\xi)$ can be expressed as

$$
y(\xi)=\mathbf{N q}^{e}
$$

If the cubic Hermitian polynomials [3] are used as the shape functions of a beam element, the shape matrix $\mathbf{N}$ of the beam element can be written as

$$
\begin{aligned}
& \mathbf{N}=\left[\begin{array}{llll}
N_{1} & N_{2} & N_{3} & N_{4}
\end{array}\right] \\
& \text { with } N_{1}=1-3(\xi / l)^{2}+2(\xi / l)^{3} \quad N_{2}=\xi\left[1-2(\xi / l)+(\xi / l)^{2}\right] \\
& N_{3}=3(\xi / l)^{2}-2(\xi / l)^{3} \quad N_{4}=\xi\left[(\xi / l)^{2}-(\xi / l)\right]
\end{aligned}
$$

where $l$ denotes the length of the beam element.

In this paper, the formulae (1)-(4) are referred to as the conventional ones for calculating the sectional forces of a Bernoulli-Euler beam under dynamic loads. The shortcomings using the formulae (1)-(4) to calculate the sectional forces of a beam element on continuously viscoelastic foundation subjected to concentrated moving loads are as follows. First, there are shortcomings using the formulae (1)-(4) to calculate the sectional forces at two nodes of a beam element. The formulae (1) and (2) for calculating the sectional forces at the left node (i.e., $\xi=0$ ) and the formulae (3) and (4) for calculating the sectional forces at the right node (i.e., $\xi=l$ ) in a beam element only consider the sectional forces induced by the nodal displacements of the corresponding beam element, because the formulae (1) and (2) with $\xi=0$ and the formulae (3) and (4) with $\xi=l$ can be expressed as the sum of the products of the corresponding element stiffness matrix and the displacements of the corresponding beam element nodes, i.e., $\mathbf{f}_{d}^{e}=\mathbf{k}_{b} \mathbf{q}^{e}$, in which, $\mathbf{f}_{d}^{e}$ denotes the sectional forces of the beam element induced by the nodal displacements of the beam element, and $\mathbf{k}_{b}$ denotes the element stiffness matrix of the beam element itself. The sectional forces of the cross-section at the nodes of element induced by the nodal accelerations and the foundation spring and damping forces acting on the corresponding beam element are not considered. In addition, the fixed-end sectional forces presented by reference [17] at the two ends of a clamped-clamped (C-C) beam element induced by moving loads acting on the beam element at time $t$ are also not considered.

Then, there exist shortcomings using the formulae (3) and (4) to calculate the sectional forces at any point A , rather than at a node, of a beam element. The formulae (3) and (4) for calculating the sectional forces of the left-hand side of cross-section at any point $\mathrm{A}$, rather than at a node, of a Bernoulli-Euler beam element and at time $t$ can be 


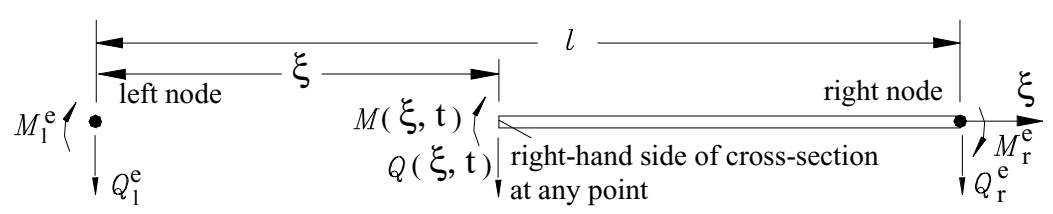

(a) Sectional forces diagram for the right-hand side of cross-section at any point

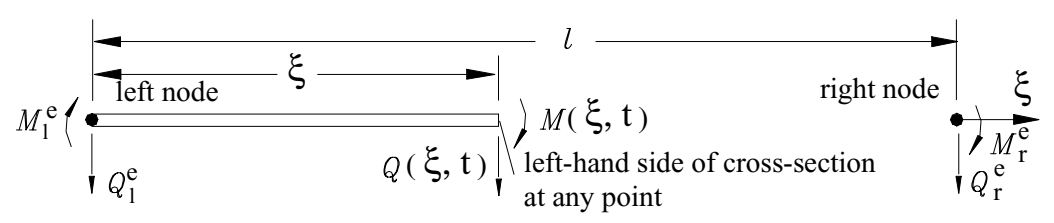

(b) Sectional forces diagram for the left-hand side of cross-section at any point

Fig. 1. Sectional forces diagram for the cross-section at any point in a Bernoulli-Euler beam element in the dynamic analysis.

written as, respectively,

$$
\begin{aligned}
\left.M(\xi, t)\right|_{\xi=\xi_{A}} & =-E I\left[\frac{6}{l^{2}} \frac{4}{l}-\frac{6}{l^{2}} \frac{2}{l}\right] \cdot \mathbf{q}^{e}+\xi_{A} \cdot E I\left[\frac{12}{l^{3}} \frac{6}{l^{2}}-\frac{12}{l^{3}} \frac{6}{l^{2}}\right] \cdot \mathbf{q}^{e} \\
& =-\left.M(\xi, t)\right|_{\xi=0}+\left.Q(\xi, t)\right|_{\xi=0} \cdot \xi_{A} \\
\left.Q(\xi, t)\right|_{\xi=\xi_{A}} & =-E I\left[\frac{12}{l^{3}} \frac{6}{l^{2}}-\frac{12}{l^{3}} \frac{6}{l^{2}}\right] \cdot \mathbf{q}^{e}=\left.Q(\xi, t)\right|_{\xi=0}
\end{aligned}
$$

The formula (7) only considers the effects of the bending moment and the shear force at the left node of the beam element, which are induced by the nodal displacements of the corresponding element, on the bending moment of the left-hand side of cross-section at the point A. In the formula (7), the effects of the fixed-end bending moment and shear force at the left end of the $\mathrm{C}-\mathrm{C}$ beam element, induced by moving loads acting on the beam element at time $t$, on the bending moment of the left-hand side of cross-section at the point A are not considered while should be considered. In addition, the effects on the bending moment of the left-hand side of cross-section at the point A are also not considered of moving loads acting on the beam element between the left node and the point A, of inertia force in the beam element between the left node and the point A, and of the foundation spring and damping forces acting on the beam element between the left node and the point $\mathrm{A}$.

Similarly, the formula (8) only considers the effect of the shear force at the left node of the beam element, which is induced by the nodal displacement of the corresponding element, on the shear force of the left-hand side of cross-section at the point A. In the formula (8), the effect of the fixed-end shear force at the left end of the C-C beam element, induced by moving loads acting on the beam element at time $t$, on the shear force of the left-hand side of cross-section at the point $\mathrm{A}$ is not considered while should be considered. In addition, the effects on the shear force of the left-hand side of cross-section at the point A are also not considered of moving loads acting on the beam element between the left node and the point A, of inertia force in the beam element between the left node and the point $\mathrm{A}$, and of the foundation spring and damping forces acting on the beam element between the left node and the point A.

This purpose of this paper is to present new finite-element formulae for calculating the sectional forces at any cross-section of a Bernoulli-Euler beam on continuously viscoelastic foundation subjected to concentrated moving loads. The new finite-element formulae can overcome the foregoing shortcomings of the conventional formulae and can improve the accuracy of calculating the sectional forces of beam. Evidently, the proposed formulae can easily degenerate into the formulae for calculating the sectional forces of a simply supported or a continuous BernoulliEuler beam subjected to concentrated moving loads, and into the formulae for calculating the sectional forces of a Bernoulli-Euler beam on Winkler foundation under static loads. 


\section{Theory and formulation}

\subsection{Fundamental assumptions}

The following assumptions are made when one establishes the formulae for calculating the sectional forces of a beam on viscoelastic foundation subjected to concentrated moving loads.

(1). Only vertical dynamic loads are considered.

(2). Axial deformations and the damping of the beam are neglected.

(3). The beam is modelled as a uniform Bernoulli-Euler beam.

(4). The viscoelastic foundation is modelled as closely spaced, independent, linear springs and viscous dampers.

(5). The cubic Hermitian polynomials [3] are used as the shape functions of a beam element.

\subsection{Equation of motion for a beam on continuously viscoelastic foundation subjected to concentrated moving loads}

Consider a uniform elastic Bernoulli-Euler beam with constant bending stiffness $E I$ resting on continuously viscoelastic foundation with stiffness coefficient $k_{w}$ and damping coefficient $c_{w}$ subjected to a number of concentrated moving loads, as shown in Fig. 2. The beam is divided into a number of finite elements with equal length of $l$. The solid circles $(\bullet)$ denote the nodes for the beam elements. Since the axial deformations of the beam are neglected, per node of a beam element has two degrees of freedom, i.e., a vertical translation and a rotation about an axis normal to the plane of the paper. According to the energy principle, the equation of motion for the beam on continuously viscoelastic foundation subjected to a number of concentrated moving loads at time $t$ can be written as

$$
\mathbf{M} \ddot{\mathbf{q}}+\mathbf{C} \dot{\mathbf{q}}+\mathbf{K q}=\sum_{i=1}^{\bar{n}} \mathbf{N}_{i}^{T} P_{i}
$$

where $\mathbf{M}, \mathbf{C}$, and $\mathbf{K}$ with $n \times n$ order are the overall mass, damping and stiffness matrices, respectively; $\ddot{\mathbf{q}}, \dot{\mathbf{q}}$, and $\mathbf{q}$ with $n \times 1$ order are the acceleration, velocity and displacement vectors, respectively; the superscript $T$ denotes transpose; $\mathbf{N}_{i}^{T}$ is the transpose of the shape functions for the beam element which are evaluated at the position of the $i$-th concentrated moving load $P_{i}$ at time $t ; P_{i}$ is the magnitude of the $i$-th concentrated moving load; $n$ is the total number of the degrees of freedom of the beam; and $\bar{n}$ is the total number of concentrated moving loads.

In Eq. (9), the overall mass matrix $\mathbf{M}$ can be obtained by assembling the element consistent mass matrix $\mathbf{m}$. Since the damping of beam itself is neglected, the overall damping matrix $\mathbf{C}$ only includes the effect of the viscous damping of foundation. $\mathbf{C}$ can be obtained by assembling the foundation element damping matrix $\mathbf{c}_{w}$ induced by viscous damping foundation supporting the beam element. The overall stiffness matrix $\mathbf{K}$ can be obtained by assembling the element stiffness matrix $\mathbf{k}_{b}$ of the beam element itself and the foundation element stiffness matrix $\mathbf{k}_{w}$ due to the elastic foundation supporting the beam element. The expressions of element matrices $\mathbf{m}, \mathbf{c}_{w}, \mathbf{k}_{b}$ and $\mathbf{k}_{w}$ are listed in the Appendix.

In addition, $\mathbf{N}_{i}$ with $1 \times n$ order in Eq. (9) can be written as

$$
\mathbf{N}_{i}=\left[\begin{array}{llllllllll}
0 & \cdots & 0 & N_{1} & N_{2} & N_{3} & N_{4} & 0 & \cdots & 0
\end{array}\right]_{\xi=\xi_{i}}
$$

where $\xi_{i}$ denotes the distance between the acting point of the $i$-th concentrated moving load $P_{i}$ and the left node of the beam element on which the load $P_{i}$ is acting at time $t$, as shown in Fig. 2 . It should be noted that $\mathbf{N}_{i}$ is a row matrix with zero entries except for those terms corresponding to two nodes of the element on which the $i$-th concentrated moving load $P_{i}$ is acting. $\mathbf{N}_{i}$ is time dependent as the load $P_{i}$ moves from one position to another within one element. As the load $P_{i}$ moves to the next element, $\mathbf{N}_{i}$ will shift in position corresponding to the degrees of freedom of the element where the load $P_{i}$ is positioned. 


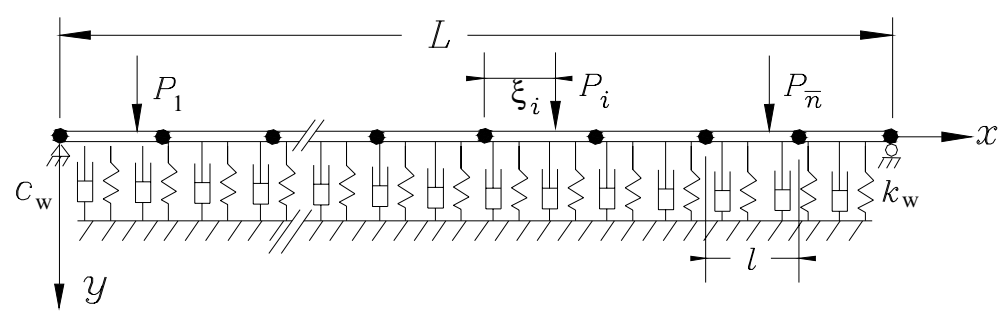

Fig. 2. Mathematical model for a uniform elastic Bernoulli-Euler beam on continuously viscoelastic foundation subjected to a number of concentrated moving loads.

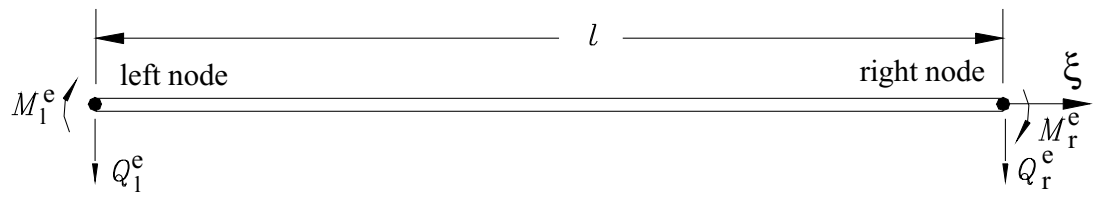

Fig. 3. Shear forces $Q_{l}^{e}$ and $Q_{r}^{e}$, and bending moments $M_{l}^{e}$ and $M_{r}^{e}$ at the cross-section of the two nodes of a typical beam element.

\subsection{Formulae for calculating the sectional forces of a beam}

By introducing the boundary conditions of the beam, Eq. (9) can be solved by the Wilson- $\theta$ method or similar methods [1], to obtain the generalized displacements, velocities and accelerations per node of the Bernoulli-Euler beam at time $t$. Then, the sectional forces at any cross-section of the beam at time $t$ can be obtained by using the following procedure.

First, let us consider how to calculate the sectional forces at the cross-section of the two nodes of a beam element. The sectional forces at the cross-section of the two nodes of a typical beam element at time $t$, as shown in Fig. 3, can be expressed as

$$
\mathbf{f}^{e}=\mathbf{m} \ddot{\mathbf{q}}^{e}+\mathbf{k}_{b} \mathbf{q}^{e}+\mathbf{c}_{w} \dot{\mathbf{q}}^{e}+\mathbf{k}_{w} \mathbf{q}^{e}+\sum_{i=1}^{h} \mathbf{f}_{i}^{0}
$$

where $\mathbf{f}^{e}$ is the sectional forces vector at the two nodes of a beam element, $\mathbf{f}^{e}=\left[\begin{array}{llll}Q_{l}^{e} & M_{l}^{e} & Q_{r}^{e} & M_{r}^{e}\end{array}\right]^{T}, Q_{l}^{e}$ and $M_{l}^{e}$ are the shear force and bending moment at the left node of the beam element, respectively; $Q_{r}^{e}$ and $M_{r}^{e}$ are the shear force and bending moment at the right node of the beam element, respectively; the positive directions of $Q_{l}^{e}, M_{l}^{e}, Q_{r}^{e}$, and $M_{r}^{e}$ are shown in Fig. 3; $\ddot{\mathbf{q}}^{e}, \dot{\mathbf{q}}^{e}$, and $\mathbf{q}^{e}$ with $4 \times 1$ order are the nodal acceleration, velocity and displacement vectors of the beam element, respectively, obtained by solving Eq. (9); $h$ is the total number of concentrated moving loads acting on the beam element at time $t$; and $\mathbf{f}_{i}^{0}$ is the fixed-end force vector [17] at the two ends of the C-C beam element induced by the $i$-th concentrated moving load $P_{i}$ acting on the beam element at time $t$. It should be noted that $\mathbf{f}_{i}^{0}$ and the equivalent nodal force vector induced by the $i$-th concentrated moving load $P_{i}$ acting on the $\mathrm{C}-\mathrm{C}$ beam element at time $t$ are equal in magnitude but opposite in direction. Thus, the expression of $\mathbf{f}_{i}^{0}$ can be written as

$$
\mathbf{f}_{i}^{0}=-\left[\begin{array}{llll}
N_{1} & N_{2} & N_{3} & N_{4}
\end{array}\right]_{\xi=\xi_{i}}^{T} \cdot P_{i}
$$

$\mathbf{f}_{i}^{0}$ will become zero vector if the $i$-th concentrated moving load $P_{i}$ acts outside the beam element.

The first and second terms on the right-hand side in Eq. (11) denote the vectors of the sectional forces at the two nodes of the beam element induced by, respectively, the nodal accelerations and the nodal displacements of the beam element. The third and fourth terms on the right-hand side denote the vectors of the sectional forces at the two nodes of the beam element induced by, respectively, the continuously damping force and the spring force under the beam element. The fifth term on the right-hand side denotes the sum of the fixed-end force vectors at the two ends of the $\mathrm{C}-\mathrm{C}$ beam element induced by $h$ concentrated moving loads acting on the beam element at time $t$.

Then, let us consider how to calculate the sectional forces at a point, rather than at a node, within a beam element. The sectional forces can be obtained by using the equilibrium condition of forces acting in the vertical direction and 


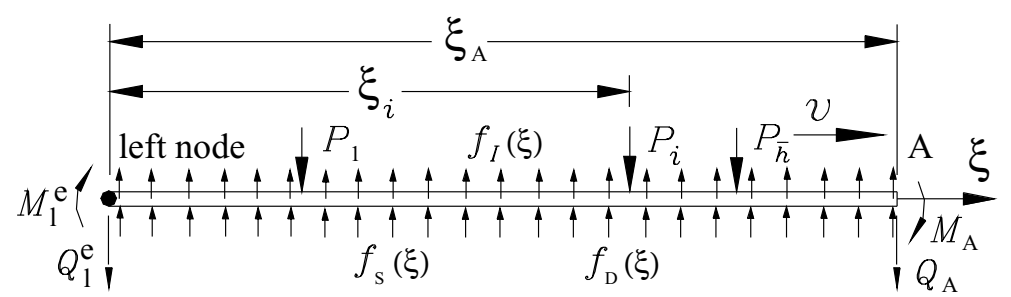

Fig. 4. Free-body diagram of portion of a typical beam element on viscoelastic foundation, in which $f(\xi)=\bar{m} \mathbf{N} \ddot{q}^{e}, f_{S}(\xi)=k_{w} \mathbf{N q}^{e}$, and $f_{D}(\xi)=c_{w} \mathbf{N} \dot{\mathbf{q}}^{e}$.

the equilibrium condition of bending moments. For example, it is assumed that point A locates at a position in the beam between two adjacent nodes, as shown in Fig. 4, and there are $\bar{h}$ concentrated moving loads between the left node of the beam element and the point $\mathrm{A}$ at time $t$. The shear force $Q_{A}$ and bending moment $M_{A}$ at the point $\mathrm{A}$ can be given by the following expression

$$
\begin{aligned}
Q_{A} & =\int_{0}^{\xi_{A}}\left(\bar{m} \mathbf{N} \ddot{\mathbf{q}}^{e}+c_{w} \mathbf{N} \dot{\mathbf{q}}^{e}+k_{w} \mathbf{N} \mathbf{q}^{e}\right) d \xi-Q_{l}^{e}-\sum_{i=1}^{\bar{h}} P_{i} \\
M_{A} & =Q_{l}^{e} \xi_{A}+\sum_{i=1}^{\bar{h}} P_{i}\left(\xi_{A}-\xi_{i}\right)-M_{l}^{e}-\int_{0}^{\xi_{A}}\left(\xi_{A}-\xi\right)\left(\bar{m} \mathbf{N} \ddot{\mathbf{q}}^{e}+c_{w} \mathbf{N} \dot{\mathbf{q}}^{e}+k_{w} \mathbf{N} \mathbf{q}^{e}\right) d \xi
\end{aligned}
$$

where $\mathbf{N}=\left[\begin{array}{llll}N_{1} & N_{2} & N_{3} & N_{4}\end{array}\right]$, and $\xi_{A}$ denotes the distance between the left node of the beam element and the point A. The positive directions of the shear force $Q_{A}$ and bending moment $M_{A}$ at the point A are shown in Figure 4. It should be noted that $Q_{l}^{e}$ and $M_{l}^{e}$ in the formulae (13a) and (14a) have been obtained by solving the formula (11).

If $\bar{m}, c_{w}$, and $k_{w}$ are constant, the formulae (13a) and (14a) can be written as, respectively,

$$
\begin{aligned}
Q_{A}= & \bar{m} \overline{\mathbf{N}}_{\xi=\xi_{A}} \ddot{\mathbf{q}}^{e}+c_{w} \overline{\mathbf{N}}_{\xi=\xi_{A}} \dot{\mathbf{q}}^{e}+k_{w} \overline{\mathbf{N}}_{\xi=\xi_{A}} \mathbf{q}^{e}-Q_{l}^{e}-\sum_{i=1}^{\bar{h}} P_{i} \\
M_{A}= & Q_{l}^{e} \xi_{A}+\sum_{i=1}^{\bar{h}} P_{i}\left(\xi_{A}-\xi_{i}\right)-M_{l}^{e}-\bar{m}\left(\xi_{A} \overline{\mathbf{N}}_{\xi=\xi_{A}}-\tilde{\mathbf{N}}_{\xi=\xi_{A}}\right) \ddot{\mathbf{q}}^{e} \\
& -c_{w}\left(\xi_{A} \overline{\mathbf{N}}_{\xi=\xi_{A}}-\tilde{\mathbf{N}}_{\xi=\xi_{A}}\right) \dot{\mathbf{q}}^{e}-k_{w}\left(\xi_{A} \overline{\mathbf{N}}_{\xi=\xi_{A}}-\tilde{\mathbf{N}}_{\xi=\xi_{A}}\right) \mathbf{q}^{e}
\end{aligned}
$$

with $\overline{\mathbf{N}}=\left[\begin{array}{llll}\bar{N}_{1} & \bar{N}_{2} & \bar{N}_{3} & \bar{N}_{4}\end{array}\right]$

$$
\begin{aligned}
& \bar{N}_{1}=\xi-\frac{\xi^{3}}{l^{2}}+\frac{1}{2} \cdot \frac{\xi^{4}}{l^{3}} \quad \bar{N}_{2}=\frac{1}{2} \cdot \xi^{2}-\frac{2}{3} \cdot \frac{\xi^{3}}{l}+\frac{1}{4} \cdot \frac{\xi^{4}}{l^{2}} \\
& \bar{N}_{3}=\frac{\xi^{3}}{l^{2}}-\frac{1}{2} \cdot \frac{\xi^{4}}{l^{3}} \quad \bar{N}_{4}=\frac{1}{4} \cdot \frac{\xi^{4}}{l^{2}}-\frac{1}{3} \cdot \frac{\xi^{3}}{l}
\end{aligned}
$$

and $\tilde{\mathbf{N}}=\left[\begin{array}{llll}\tilde{N}_{1} & \tilde{N}_{2} & \tilde{N}_{3} & \tilde{N}_{4}\end{array}\right]$

$$
\begin{aligned}
& \tilde{N}_{1}=\frac{1}{2} \cdot \xi^{2}-\frac{3}{4} \cdot \frac{\xi^{4}}{l^{2}}+\frac{2}{5} \cdot \frac{\xi^{5}}{l^{3}} \quad \tilde{N}_{2}=\frac{1}{3} \cdot \xi^{3}-\frac{1}{2} \cdot \frac{\xi^{4}}{l}+\frac{1}{5} \cdot \frac{\xi^{5}}{l^{2}} \\
& \tilde{N}_{3}=\frac{3}{4} \cdot \frac{\xi^{4}}{l^{2}}-\frac{2}{5} \cdot \frac{\xi^{5}}{l^{3}} \quad \tilde{N}_{4}=\frac{1}{5} \cdot \frac{\xi^{5}}{l^{2}}-\frac{1}{4} \cdot \frac{\xi^{4}}{l}
\end{aligned}
$$

It should be pointed out that the formula (11) only calculates the sectional forces at two nodes of a beam element and the formulaes (13) and (14) can calculate the sectional forces at any point in a beam element except two nodes. By 


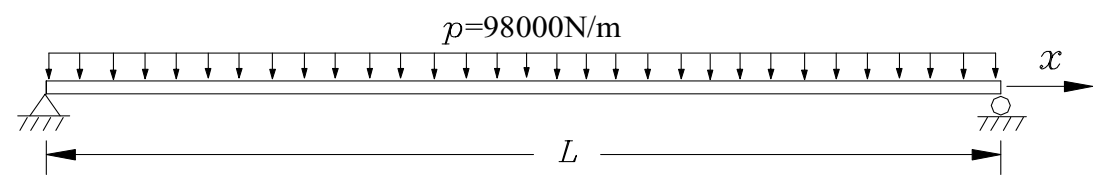

Fig. 5. A simply supported beam under uniformly distributed static load.

using the formulae (11), (13) and (14), one can obtain the sectional forces at any cross-section of a Bernoulli-Euler beam on continuously viscoelastic foundation subjected to a number of concentrated moving loads at time $t$.

Furthermore, for the "static" problem, one has $\ddot{\mathbf{q}}^{e}=\dot{\mathbf{q}}^{e}=0$, and the formulae (11), (13) and (14) reduce to, respectively,

$$
\begin{aligned}
& \mathbf{f}^{e}=\left(\mathbf{k}_{b}+\mathbf{k}_{w}\right) \mathbf{q}^{e}+\sum_{i=1}^{h} \mathbf{f}_{i}^{0} \\
& Q_{A}=\int_{0}^{\xi_{A}} k_{w} \mathbf{N q}^{e} d \xi-Q_{l}^{e}-\sum_{i=1}^{\bar{h}} P_{i} \\
& M_{A}=Q_{l}^{e} \xi_{A}+\sum_{i=1}^{\bar{h}} P_{i}\left(\xi_{A}-\xi_{i}\right)-M_{l}^{e}-\int_{0}^{\xi_{A}}\left(\xi_{A}-\xi\right) k_{w} \mathbf{N} \mathbf{q}^{e} d \xi
\end{aligned}
$$

If $k_{w}$ is constant, the formulae (16a) and (17a) can be written as, respectively,

$$
\begin{aligned}
Q_{A} & =k_{w} \overline{\mathbf{N}}_{\xi=\xi_{A}} \mathbf{q}^{e}-Q_{l}^{e}-\sum_{i=1}^{\bar{h}} P_{i} \\
M_{A} & =Q_{l}^{e} \xi_{A}+\sum_{i=1}^{\bar{h}} P_{i}\left(\xi_{A}-\xi_{i}\right)-M_{l}^{e}-k_{w}\left(\xi_{A} \overline{\mathbf{N}}_{\xi=\xi_{A}}-\tilde{\mathbf{N}}_{\xi=\xi_{A}}\right) \mathbf{q}^{e}
\end{aligned}
$$

In addition, if $k_{w}=0$ and $c_{w}=0$ are used in the formulae (11), (13) and (14), then the revised formulae of (11), (13) and (14) can be used to calculate the sectional forces at any cross-section of a simply supported or a continuous Bernoulli-Euler beam subjected to a number of concentrated moving loads.

\section{Numerical examples}

Five numerical examples including static and dynamic analyses are chosen to illustrate the application of the proposed formulae. In the finite element analysis, the equation of motion for the following dynamic analyses will be solved by means of the Wilson- $\theta$ method with $\theta=1.4$.

\subsection{Example 1. A simply supported beam under uniformly distributed static load}

Figure 5 shows a simply supported beam under uniformly distributed static load $p=9.8 \times 10^{4} \mathrm{~N} / \mathrm{m}$. The parameters of the beam taken from reference [4] are: length $L=0.2 \mathrm{~m}$ and cross-section area $=2.0 \times 10^{-4} \mathrm{~m}^{2}$ ( $0.02 \mathrm{~m}$ deep $\times 0.01 \mathrm{~m}$ wide) and $E=9.8 \times 10^{10} \mathrm{~N} / \mathrm{m}^{2}$.

For the case of a simply supported beam under uniformly distributed static load, the formula (11) for calculating the sectional forces at the two nodes of a beam element can be revised as

$$
\mathbf{f}^{e}=\mathbf{k}_{b} \mathbf{q}^{e}+\mathbf{f}^{0}
$$




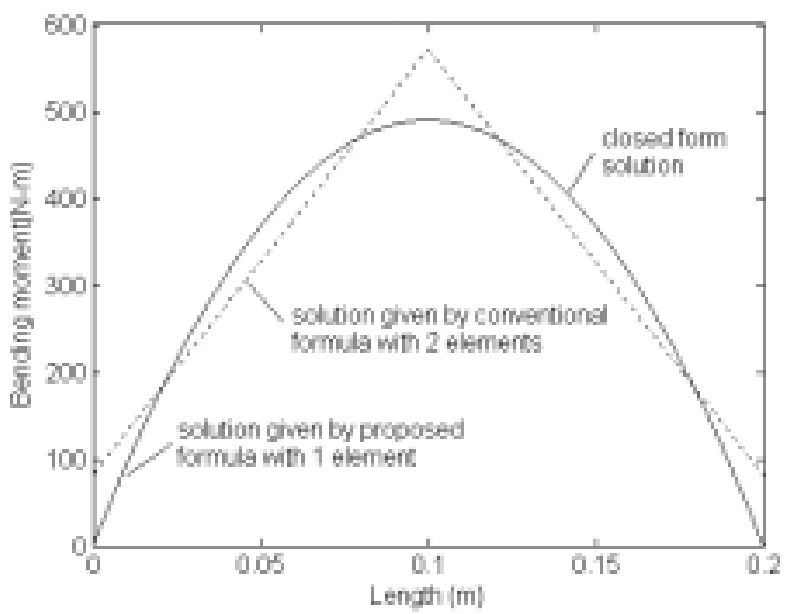

Fig. 6. Bending moment diagrams in a simply supported beam under uniformly distributed load.

where $\mathrm{f}^{0}$ denotes the fixed-end force vector at the two ends of the $\mathrm{C}-\mathrm{C}$ beam element induced by uniformly distributed static load, which can be written as

$$
\mathbf{f}^{0}=-\left[\begin{array}{llll}
p L / 2 & p L^{2} / 12 \quad p L / 2 & -p L^{2} / 12
\end{array}\right]^{T}
$$

The formulaes (13) and (14) for calculating the sectional forces at a point A, rather than at a node, in a beam element can be revised as

$$
\begin{aligned}
& Q_{A}=-Q_{l}^{e}-p \cdot \xi_{A} \\
& M_{A}=Q_{l}^{e} \xi_{A}+\int_{0}^{\xi_{A}}\left(\xi_{A}-\xi\right) p d \xi-M_{l}^{e}=Q_{l}^{e} \xi_{A}+\frac{1}{2} p \cdot \xi_{A}^{2}-M_{l}^{e}
\end{aligned}
$$

Bending moment diagrams and shear force diagrams of the simply supported beam have been plotted in Figs 6 and 7, respectively, including closed form solutions and finite element solutions given by the proposed formulae with 1 element and by the conventional formulae [4] with 2 elements of equal length. It can be seen from Figs 6 and 7 that the finite element solutions given by the proposed formulae with 1 element are the same as the closed form solutions; however, the same agreement cannot be found between the finite element solutions given by the conventional formulae with 2 elements and the closed form solutions. The reasons are as follows.

The conventional formulae used in reference [4] is

$$
\mathrm{f}^{e}=\mathbf{k}_{b} \mathbf{q}^{e}
$$

Compared with the proposed formula (18), the conventional formula (22) neglects $\mathbf{f}^{0}$ to calculate the sectional forces at two nodes of a beam element. In addition, compared with the proposed formulaes (20) and (21), the conventional formula (22) does not consider (i) the sectional forces at point A contributed by the fixed-end force at the left end of the $\mathrm{C}-\mathrm{C}$ beam element induced by the uniformly distributed load acting on the beam element, and (ii) the sectional forces at point A induced by the uniformly distributed load acting on the beam element between the left node and the point $\mathrm{A}$, when the conventional formula (22) is used to calculate the sectional forces at the point $\mathrm{A}$ in a beam element.

\subsection{Example 2. A beam with free ends on Winkler foundation under a concentrated static load}

Consider a uniform Bernoulli-Euler beam with free ends resting on Winkler foundation under a concentrated static load $P=10,000 \mathrm{~N}$ acting on the beam midpoint, as shown in Fig. 8. The parameters in this study are given: $E$ (beam elastic modulus $)=9,100 \times 10^{6} \mathrm{~N} / \mathrm{m}^{2}, I($ moment of inertia of beam $)=7.326 \times 10^{-5} \mathrm{~m}^{4}, L$ (beam length $)=$ 


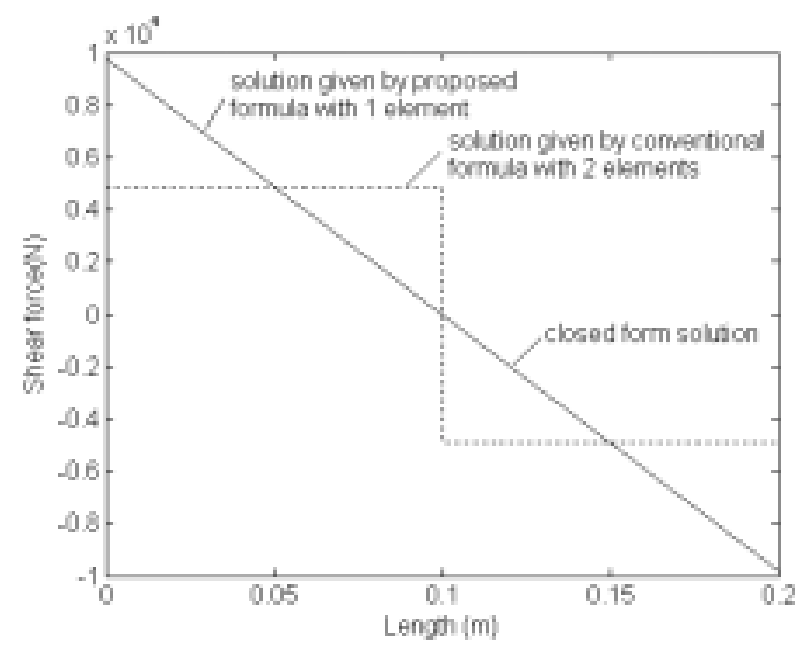

Fig. 7. Shear force diagrams in a simply supported beam under uniformly distributed load.

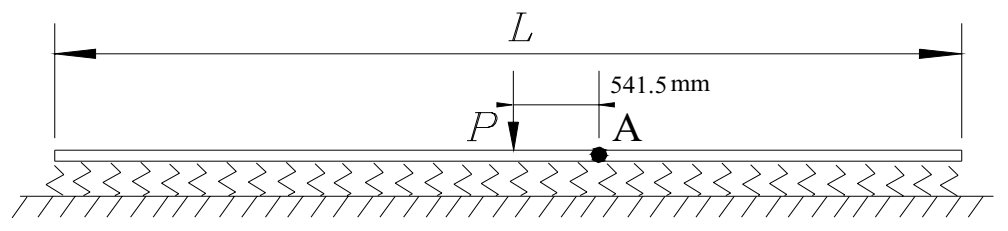

Fig. 8. A uniform Bernoulli-Euler beam with free end resting on Winkler foundation under a concentrated static load.

$18.05 \mathrm{~m}$, and $k_{w}$ (stiffness coefficient of Winkler foundation) $=4.0 \times 10^{6} \mathrm{~N} / \mathrm{m}^{2}$. These parameters of this example are taken from reference [7].

Table 1 reports the ratios of the numerical solutions to the exact solutions of the sectional forces at the point A , with the numerical solutions given, respectively, by the proposed formulaes (16b) and (17b), and by the conventional formula (22) adopted in reference [7] using the cubic Hermitian polynomials as the shape functions of a beam element. The distance between the point $\mathrm{A}$ and the beam midpoint is $0.5415 \mathrm{~m}$, as shown in Fig. 8. The exact solutions can be obtained using the formulae presented in reference [21]. It should be noted that exact solutions are not dependent on the number of elements. It can be seen from Table 1 that the bending moment and the shear force given by the proposed formulaes (16b) and (17b) are nearly exact if 20 elements are used for the beam length $L$. The error of the shear force is 3 per cent and the error of the bending moment is less than 8 per cent if only 10 elements are used. However, the same agreement cannot be found for the results given by the conventional formula (22) adopted in reference [7]. This is due to the shortcomings of the conventional formulae.

\subsection{Example 3. A simply supported beam subjected to a concentrated moving load}

Consider a uniform simply supported Bernoulli-Euler beam with a span $L$ of $20 \mathrm{~m}$ subjected to a concentrated moving load $P=215.6 \mathrm{kN}$ with constant speed $v=60 \mathrm{~m} / \mathrm{s}$ from the left end to the right end of the beam. The beam parameters are: $E=2.9430 \times 10^{10} \mathrm{~N} / \mathrm{m}^{2}, I=3.81 \mathrm{~m}^{4}$, and $\bar{m}=34,088 \mathrm{~kg} / \mathrm{m}$.

In the finite element analysis, the beam is modelled as 2, 6 and 10 elements with equal length, respectively, and time interval $0.005 \mathrm{~s}$ is adopted. The bending moments at the midpoint cross-section of beam given by the revised proposed formula (11), i.e., after deleting the third and fourth terms of right hand side in formula (11), with 2, 6 and 10 elements have been plotted in Fig. 9, along with the analytical solution given by the following Eq. (23) taken from Timoshenko et al. [22] with $i=1-2000$.

$$
M(x, t)=-E I \frac{\partial^{2} y(x, t)}{\partial x^{2}}
$$


Table 1

Ratios of the numerical solutions to the exact solutions of the sectional forces at the point A in Example 2 with the numerical solutions given, respectively, by the proposed formulae and by the conventional formulae in reference [7]

\begin{tabular}{lcccccc}
\hline Grade of mesh (elements of equal length) & \multicolumn{2}{c}{ Bending moment } & & \multicolumn{2}{c}{ Shear force } \\
\cline { 2 - 3 } \cline { 6 - 7 } & Present & Ref. [7] & & Present & Ref. [7] \\
\hline 10 elements & 0.9243 & 1.8109 & & 1.0300 & 0.5683 \\
20 elements & 0.9920 & 1.3908 & & 0.9983 & 1.2046 \\
40 elements & 0.9996 & 0.9951 & & 1.0000 & 0.7720 \\
80 elements & 1.0000 & 1.0240 & & 1.0000 & 0.9603 \\
160 elements & 1.0000 & 0.9994 & & 1.0000 & 1.0644 \\
\hline
\end{tabular}

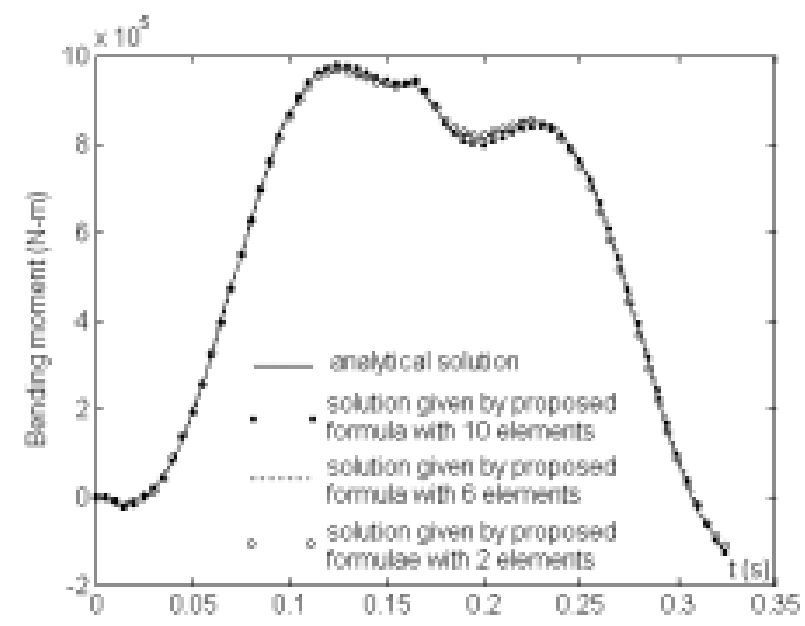

Fig. 9. Time histories for bending moment at the midpoint cross-section of beam with analytical solution, and the solutions given by the proposed formula with 2, 6 and 10 elements.

with

$$
\begin{aligned}
y(x, t) & =\frac{2 P L^{3}}{\bar{m} \pi^{2}} \sum_{i=1}^{\infty} \frac{\sin (i \pi x / L) \sin (i \pi v t / L)}{i^{2}\left(i^{2} \pi^{2} a^{2}-v^{2} L^{2}\right)}-\frac{2 P L^{4} v}{\bar{m} \pi^{3} a} \sum_{i=1}^{\infty} \frac{\sin (i \pi x / L) \sin \left(i^{2} \pi^{2} a t / L^{2}\right)}{i^{3}\left(i^{2} \pi^{2} a^{2}-v^{2} L^{2}\right)} \\
a^{2} & =\frac{E I}{\bar{m}}
\end{aligned}
$$

From Fig. 9, good agreement has been achieved between the present solution with 2, 6 and 10 elements, respectively, and the analytical solution.

The bending moments at the midpoint cross-section of beam given by the revised proposed formula (11) and the conventional formula (1) with 2, 6 and 10 elements have been plotted in Figs 10-12, respectively. It can be seen that the difference between the solution given by the revised proposed formula (11) and that given by the conventional formula (1) increases with the increase of length of element. The reason why there is the difference between the solution given by the revised proposed formula (11) and that given by the conventional formula (1) is that the fixed-end bending moment at the left end of the $(N / 2+1)$-th $\mathrm{C}-\mathrm{C}$ beam element induced by the load acting on the $(N / 2+1)$-th beam element is not considered in the conventional formula, in which $N$ denotes the total number of beam elements.

\subsection{Example 4. A simply supported beam resting on Winkler foundation subjected to a stationary pulsating concentrated load}

Let us consider a simply supported uniform Bernoulli-Euler beam resting on Winkler foundation subjected to a stationary pulsating concentrated load of $P(t)=\bar{P} \sin \omega_{e} t$ acting at a distance $x_{1}$ from the left end point of the 


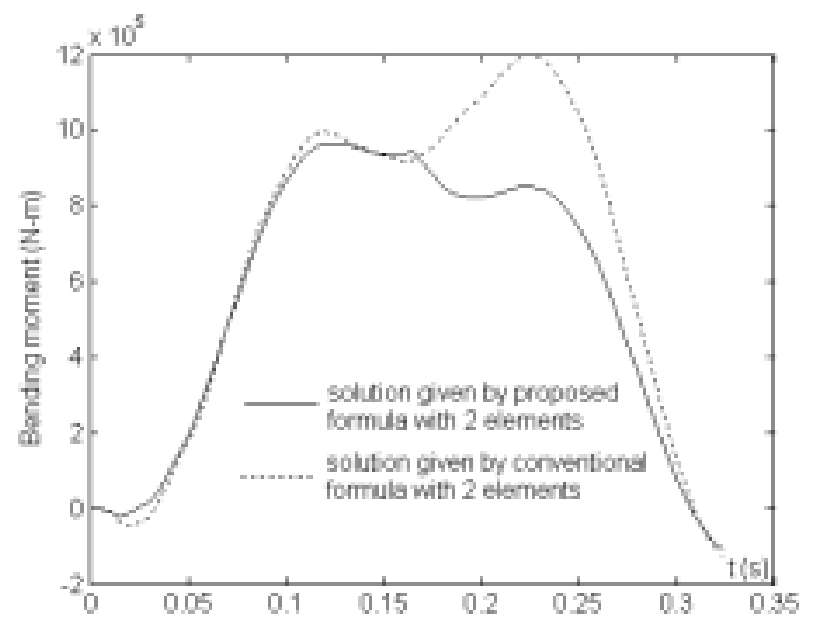

Fig. 10. Time histories for bending moment at the midpoint cross-section of beam with the solutions given by the proposed formula and by the conventional formula with 2 elements.

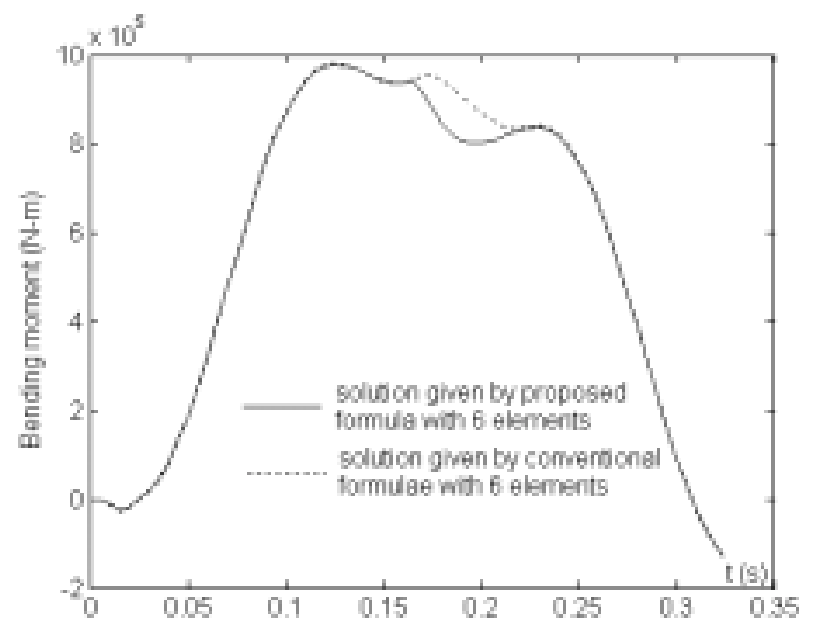

Fig. 11. Time histories for bending moment at the midpoint cross-section of beam with the solutions given by the proposed formula and by the conventional formula with 6 elements.

beam. The expressions of the exact vertical displacement $y(x, t)$ and bending moment $M(x, t)$ of the beam taken from Timoshenko et al. [22] are as follows

$$
\begin{aligned}
& y(x, t)=\frac{2 \bar{P} L^{3}}{\bar{m}} \sum_{i=1}^{\infty} \sin (i \pi x / L) \sin \left(i \pi x_{1} / L\right)\left[\frac{\sin \omega_{e} t}{\pi^{4} a^{2}\left(i^{4}+\mu^{4}\right)-\omega_{e}^{2} L^{4}}-\frac{\omega_{e} \sin \omega_{i} t}{L^{4} \omega_{i}\left(\omega_{i}^{2}-\omega_{e}^{2}\right)}\right] \\
& M(x, t)=-E I \frac{\partial^{2} y(x, t)}{\partial x^{2}}
\end{aligned}
$$

with

$$
\begin{aligned}
& \omega_{i}=a\left(\frac{\pi}{L}\right)^{2} \sqrt{i^{4}+\mu^{4}} \quad i=1,2, \ldots \\
& a^{2}=\frac{E I}{\bar{m}}
\end{aligned}
$$




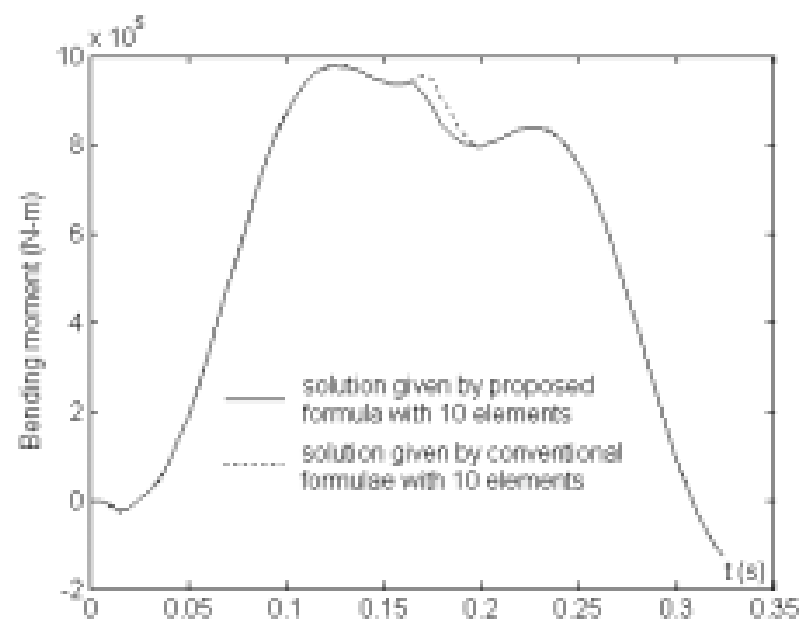

Fig. 12. Time histories for bending moment at the midpoint cross-section of beam with the solutions given by the proposed formula and by the conventional formula with 10 elements.

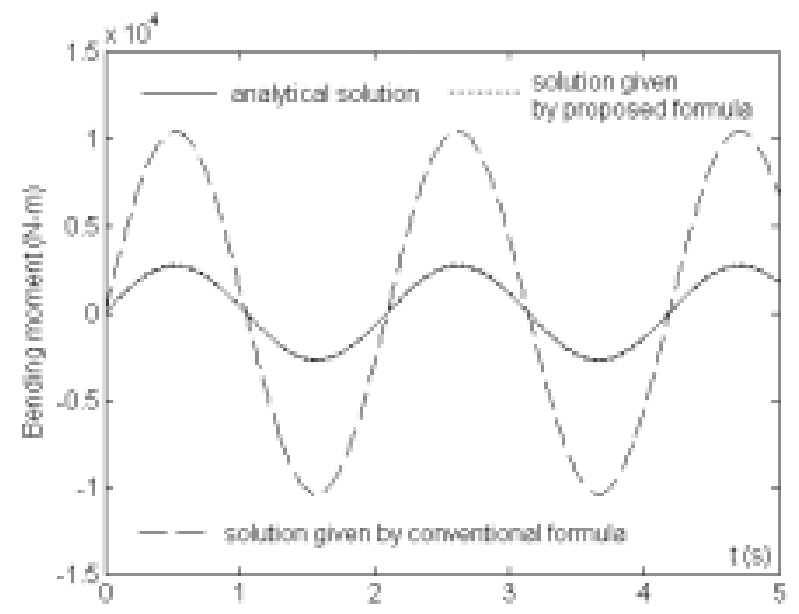

Fig. 13. Time histories for bending moment at the midpoint cross-section of beam in Example 4 .

$$
\mu^{4}=\left(\frac{L}{\pi}\right)^{4} \frac{k_{w}}{E I}
$$

The parameters in this study are: $E=20.6 \times 10^{10} \mathrm{~N} / \mathrm{m}^{2}, I=2.037 \times 10^{-5} \mathrm{~m}^{4}, \bar{m}=50 \mathrm{~kg} / \mathrm{m}, L=100 \mathrm{~m}, x_{1}=$ $50.5 \mathrm{~m}, k_{w}=3.73 \times 10^{7} \mathrm{~N} / \mathrm{m}^{2}, \bar{P}=98,000 \mathrm{~N}$, and $\omega_{e}=3 \mathrm{rad} / \mathrm{s}$.

The time histories for the bending moment at the midpoint cross-section of beam are shown in Fig. 13, where the solid, the dotted and the dashed lines denote the time histories with the analytical solution given by Eq. (26) with $i=1-2000$, with the finite element solutions (with equal element length $1.0 \mathrm{~m}$ and time interval $0.01 \mathrm{~s}$ ) given by the revised proposed formula (11), i.e., after deleting the third term of right hand side in formula (11), and given by the conventional formula (1), respectively. It is evident that the time histories with the finite element solution given by the revised proposed formula (11) are very close to those with the analytical solution given by Eq. (26). However, the difference between the time histories with the finite element solution given by the conventional formula (1) and those with the analytical solution given by Eq. (26) is high. This is due to the shortcomings mentioned in Section 1 of Introduction. 
Table 2

Present solutions (with 100 elements of equal length) and analytical solutions of maximum bending moments at the midpoint cross-section of beam, and the ratios of the solution given by the proposed formula to the corresponding analytical solution subjected to a moving load with various speeds

\begin{tabular}{lcccccc}
\hline Speed $(\mathrm{m} / \mathrm{s})$ & 10 & 30 & 50 & 70 & 90 & 110 \\
\hline Present solution $(\mathrm{N}-\mathrm{m})$ & 19859 & 19878 & 19915 & 19965 & 20036 & 20129 \\
Analytical solution $(\mathrm{N}-\mathrm{m})$ & 20068 & 20084 & 20116 & 20164 & 20229 & 20311 \\
Ratio of present solution to analytical one & 0.9896 & 0.9897 & 0.9900 & 0.9901 & 0.9905 & 0.9910 \\
\hline
\end{tabular}

\subsection{Example 5. A simply supported beam resting on viscoelastic foundation subjected to a concentrated moving load}

A simply supported uniform Bernoulli-Euler beam resting on continuously viscoelastic foundation subjected to a concentrated moving load $P=98,000 \mathrm{~N}$ with constant speed from the left end to the right end of the beam is studied in this example. The damping coefficient of foundation, $c_{w}$, is defined by a non-dimensional parameter [6] (the ratio between actual damping and critical damping) given by $\beta=\frac{c_{w}}{2 \bar{m}} \sqrt{\bar{m} / k_{w}} \cdot \beta=0.1$ is adopted in this example. Other parameters for the beam and the foundation are the same as those in Example 4. Initially, the beam is at rest, and the moving load is at the left end of the beam. In the present analysis, 1000 equal time steps for the case of every speed of the moving load are adopted.

The maximum bending moments at the midpoint cross-section of beam given by the proposed formula (11) using 100 elements with equal length under the moving load with various speeds have been reported in Table 2, along with the analytical solutions given by the following Eq. (27) taken from Fr ýba [9]

$$
M(\alpha)=\frac{1}{\left(1-\alpha^{2}\right)^{1 / 2}\left[1+\frac{1}{2} \alpha^{2} \beta^{2} /\left(1-\alpha^{2}\right)^{2}\right]} \cdot \frac{P}{4 \lambda} \text { Case with } \alpha<1 \text { and } \beta<<1
$$

with

$$
\begin{aligned}
& \alpha=\frac{v}{2 \lambda} \sqrt{\bar{m} / E I} \\
& \lambda=\left(k_{w} / 4 E I\right)^{1 / 4}
\end{aligned}
$$

It is observed from Table 2 that the present solution agrees well with the corresponding analytical one, and the difference between the present solution and the analytical one is about 1 per cent.

The maximum bending moments at the midpoint cross-section of beam given by the proposed formula (11) with $40,60,80,100,120,140$, and 160 elements and constant speed $v=50 \mathrm{~m} / \mathrm{s}$ have been reported in Table 3, along with those given by the conventional formula (1) at the corresponding time when the maximum bending moment is achieved by the proposed formula (11), i.e., when the moving load is acting on the beam midpoint. In addition, the two results have been plotted in Fig. 14. From Table 3 and Fig. 14, one can obtain that the difference between the solution given from the proposed formula (11) and the analytical solution given by Eq. (27) is low, for example, the difference between the two results is 4.8 per cent even though 40 elements (i.e., element length of $2.5 \mathrm{~m}$ ) have been used for the beam; however, the difference between the solution given by the conventional formula (1) and the analytical solution given by Eq. (27) is high, for example, the differences between the two results are 68.1 and 9.8 per cent, respectively, when 40 and 160 elements have been used for the beam. This is due to the shortcomings of the conventional formula mentioned Section 1 of Introduction.

It should be pointed out that Eq. (27) is based on the following assumption [9], i.e., an infinite beam on a Winkler foundation is subjected to a constant load $P$ moving from infinity to infinity at constant speed $v$. The differential equation of the vertical vibration of the beam given by reference [9] is written as

$$
\frac{d^{4} y(s)}{d s^{4}}+4 \alpha^{2} \frac{d^{2} y(s)}{d s^{2}}-8 \alpha \beta_{1} \frac{d y(s)}{d s}+4 y(s)=8 \bar{\delta}(s)
$$

with 
Table 3

Maximum bending moments at the midpoint cross-section of beam given by the proposed formula and those given by the conventional formula with various element numbers subjected to a moving load with constant speed $v=50 \mathrm{~m} / \mathrm{s}$

\begin{tabular}{|c|c|c|c|c|c|c|c|}
\hline $\begin{array}{l}\text { Grade of mesh (elements } \\
\text { of equal length) }\end{array}$ & 40 elements & 60 elements & 80 elements & 100 elements & 120 elements & 140 elements & 160 elements \\
\hline $\begin{array}{l}\text { Solution given by the } \\
\text { proposed formula }(\mathrm{N}-\mathrm{m})\end{array}$ & 21079 & 19991 & 19895 & 19915 & 19912 & 19947 & 19933 \\
\hline $\begin{array}{l}\text { Solution given by the } \\
\text { conventional formula } \\
(\mathrm{N}-\mathrm{m})\end{array}$ & 6416 & 11020 & 13998 & 15804 & 16904 & 17663 & 18142 \\
\hline
\end{tabular}

Analytical solution is $20116 \mathrm{~N}-\mathrm{m}$.

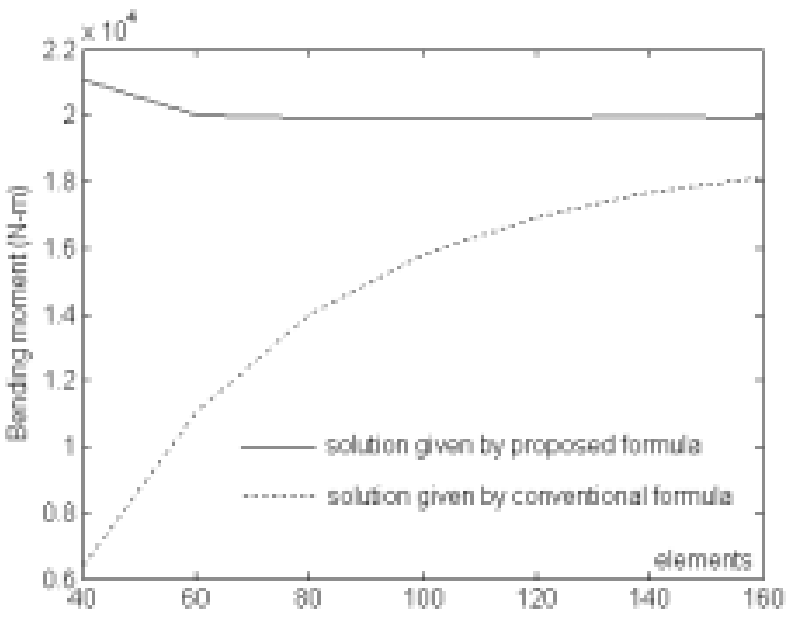

Fig. 14. Maximum bending moments at the midpoint cross-section of beam given by the proposed formula and those given by the conventional formula with various element numbers subjected to a moving load with constant speed $v=50 \mathrm{~m} / \mathrm{s}$.

$$
\begin{aligned}
s & =\lambda(x-v t) \\
\beta_{1} & =\omega_{b} \sqrt{\bar{m} / k_{w}} \\
\bar{\delta}(s) & =\delta(x) / \lambda
\end{aligned}
$$

where $\omega_{b}$ denotes circular frequency of damping of the beam, $v$ is moving speed of the load, and $\delta(x)$ is Dirac delta function.

The reasons why Eq. (27) can be used as the analytical solution of this example are as follows. On the one hand, if $\beta_{1}$ in Eq. (28) is replaced by $\beta=\frac{c_{w}}{2 \bar{m}} \sqrt{\bar{m} / k_{w}}$, then the modified Eq. (28) is the differential equation of the vertical vibration of an infinite beam on a continuously viscoelastic foundation subjected to a constant load $P$ moving from infinity to infinity at constant speed $v$. On the other hand, the displacements and sectional forces of the beam on elastic foundation decline to zero very fast as the distance from point load increases [21]. Therefore, one may choose a beam with finite length to take the place of an infinite beam.

\section{Concluding remarks}

This paper has presented new finite-element formulae overcoming the shortcomings of the conventional formulae to calculate the sectional forces at any cross-section of a Bernoulli-Euler beam on continuously viscoelastic foundation subjected to concentrated moving loads. The proposed formulae can easily degenerate into the formulae for calculating the sectional forces of a simply supported or a continuous Bernoulli-Euler beam subjected to concentrated moving loads, and into the formulae for calculating the sectional forces of a Bernoulli-Euler beam on Winkler foundation under static loads. Five numerical examples including static and dynamic analyses are chosen to illustrate 
the application of the proposed formulae. Numerical results show: (i) compared with the conventional formulae, the proposed formulae can improve the calculation accuracy of the sectional forces of beam; (ii) to calculate the sectional forces at the cross-section of the two nodes of a beam element, one should use the proposed formula (11), not the conventional formulae (1), (2) or (3), (4); (iii) to calculate the sectional forces at point within a beam element rather than at a node, one should use the proposed formulae (13), (14), not the conventional formulae (1), (2) or (3), (4).

\section{Acknowledgement}

The author would like to thank Prof. Daniel J. Inman, Editor-in-Chief of Shock and Vibration, Dr. Sondipon Adhikari, Associate Editor of Shock and Vibration and two anonymous reviewers for their valuable and helpful comments.

\section{Appendix}

The expression of the element consistent mass matrix $\mathbf{m}$ with $4 \times 4$ order [2] used in the Eq. (11) is

$$
\mathbf{m}=\frac{\bar{m} l}{420}\left[\begin{array}{cccc}
156 & 22 l & 54 & -13 l \\
& 4 l^{2} & 13 l & -3 l^{2} \\
& & 156 & -22 l \\
\text { symm. } & & & 4 l^{2}
\end{array}\right]
$$

where $\bar{m}$ is the mass per unit length of beam.

The expression of the element stiffness matrix $\mathbf{k}_{b}$ with $4 \times 4$ order [2] used in the Eq. (11) is

$$
\mathbf{k}_{b}=E I\left[\begin{array}{cccl}
12 / l^{3} & 6 / l^{2} & -12 / l^{3} & 6 / l^{2} \\
& 4 / l & -6 / l^{2} & 2 / l \\
& & 12 / l^{3} & -6 / l^{2} \\
\text { symm. } & & & 4 / l
\end{array}\right]
$$

The expressions of the foundation element stiffness matrix $\mathbf{k}_{w}$ and damping matrix $\mathbf{c}_{w}$ used in the Eq. (11) are similar in form to the element consistent mass matrix $\mathbf{m}$ of Eq. (A-1). $\mathbf{k}_{w}$ can be obtained by simply replacing $\bar{m}$ by $k_{w}$ in the element consistent mass matrix $\mathbf{m}$. Similarly, $\mathbf{c}_{w}$ can also be obtained by simply replacing $\bar{m}$ by $c_{w}$ in the element consistent mass matrix $\mathbf{m}$.

\section{References}

[1] K.J. Bathe and E.L. Wilson, Numerical Methods in Finite Element Analysis, Prentice-Hall, Englewood Cliffs, New Jersey, 1976.

[2] R.W. Clough and J. Penzien, Dynamics of Structures, (2nd edition), McGraw-Hill, New York, 1993.

[3] R.D. Cook, Concepts and Applications of Finite Element Analysis, (2nd edition), Wiley, New York, 1981.

[4] C.S. Desai, Elementary Finite Element Method, Prentice-Hall, Inc., Englewood Cliffs, New Jersey, 1979, $181-185$.

[5] D.G. Duffy, The response of an infinite railroad track to a moving vibrating mass, Journal of Applied Mechanics, ASME 57(1) (1990), 66-73.

[6] C. Esveld, Modern Railway Track, (2nd edition), MRT-Productions, Zaltbommel, ISBN 90-800324-3-3, 2001.

[7] Z. Feng and R.D. Cook, Beam element on two-parameter elastic foundations, Journal of Engineering Mechanics ASCE 109(6) (1983), 1390-1402.

[8] A.L. Florence, Traveling force on a Timoshenko beam, Journal of Applied Mechanics ASME 32(2) (1965), 351-358.

[9] L. Frýba, Vibration of Solids and Structures under Moving Loads, (3rd edition), Academia, Prague, 1999.

[10] L. Frýba, S. Nakagiri and N. Yoshikawa, Stochastic finite elements for a beam on a random foundation with uncertain damping under a moving force, Journal of Sound and Vibration 163(1) (1993), 31-45.

[11] A. Garinei, Vibrations of simple beam-like modelled bridge under harmonic moving loads, International Journal of Engineering Science 44(11-12) (2006), 778-787.

[12] J.T. Kenney, Steady-state vibrations of beam on elastic foundation for moving load, Journal of Applied Mechanics, ASME 21(4) (1954), $359-364$. 
[13] T. Kocaturk and M. Simsek, Vibration of viscoelastic beams subjected to an eccentric compressive force and a concentrated moving harmonic force, Journal of Sound and Vibration 291(1-2) (2006), 302-322.

[14] P. Lou, G.L. Dai and Q.Y. Zeng, Dynamic analysis of a timoshenko beam subjected to moving concentrated forces using the finite element method, Shock and Vibration, in press.

[15] S.P. Patil, Natural frequencies of an infinite beam on a simple inertial foundation model, International Journal of Solids and Structures 23(12) (1987), 1615-1623.

[16] S.P. Patil, Response of infinite railway track to vibrating mass, Journal of Engineering Mechanics, ASCE 114(4) (1988), 688-703.

[17] R.L. Sack, Structural Analysis, McGraw-Hill Book Company, New York, 1984.

[18] V.N. Shah, R.D. Cook and T.C. Huang, Loads moving on beam supported by layered elastic foundation, Journal of Mechanical Design, ASME 102(2) (1980), 295-302.

[19] D. Thambiratnam and Y. Zhuge, Dynamic analysis of beams on an elastic foundation subjected to moving loads, Journal of Sound and Vibration 198(2) (1996), 149-169.

[20] S. Timoshenko, Method of analysis of statical and dynamical stresses in rails, Proceedings of the Second International Congress for Applied Mechanics, Zurich, Swiss, 1926, 407-418.

[21] S. Timoshenko, Strength of Materials Part II Advance Theory and Problems, VanNostrand Reinhold Company Ltd, London, 1956.

[22] S. Timoshenko, D.H. Young and W. Weaver, Jr., Vibration Problems in Engineering, (4th edition), Wiley, New York, 1974.

[23] J.J. Wu, Dynamic analysis of an inclined beam due to moving loads, Journal of Sound and Vibration 288(1-2) (2005), 107-131.

[24] J.J. Wu, A.R. Whittaker and M.P. Cartmell, The use of finite element techniques for calculating the dynamic response of structures to moving loads, Computers and Structures 78(6) (2000), 789-799.

[25] J.S. Wu and P.Y. Shin, Dynamic responses of railway and carriage under the high-speed moving loads, Journal of Sound and Vibration 236(1) (2000), 61-87.

[26] J.S. Wu and P.Y. Shin, The dynamic behaviour of a finite railway under the high-speed multiple moving forces by using finite element method, Communications in Numerical Methods in Engineering 16(12) (2000), 851-866. 

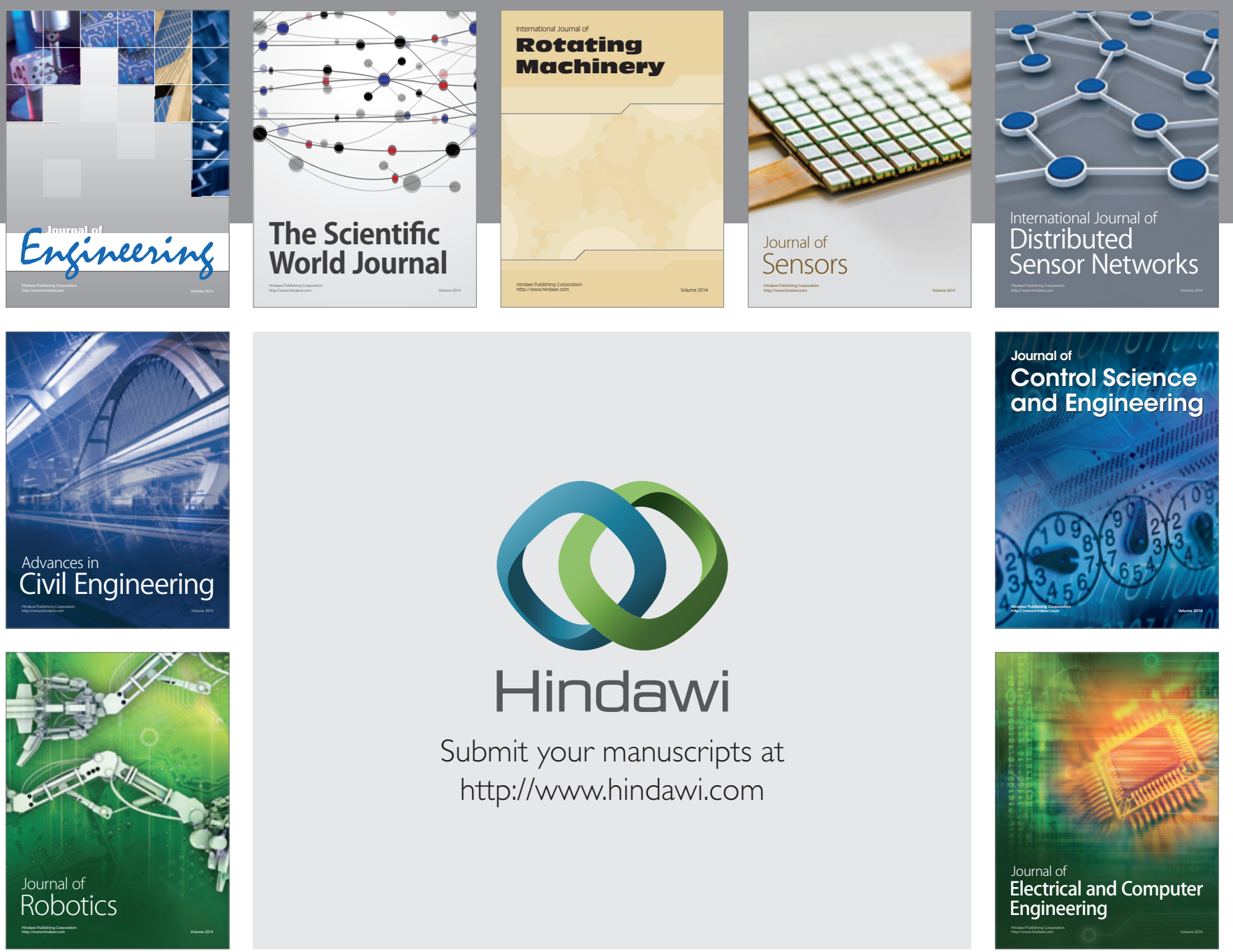

Submit your manuscripts at

http://www.hindawi.com
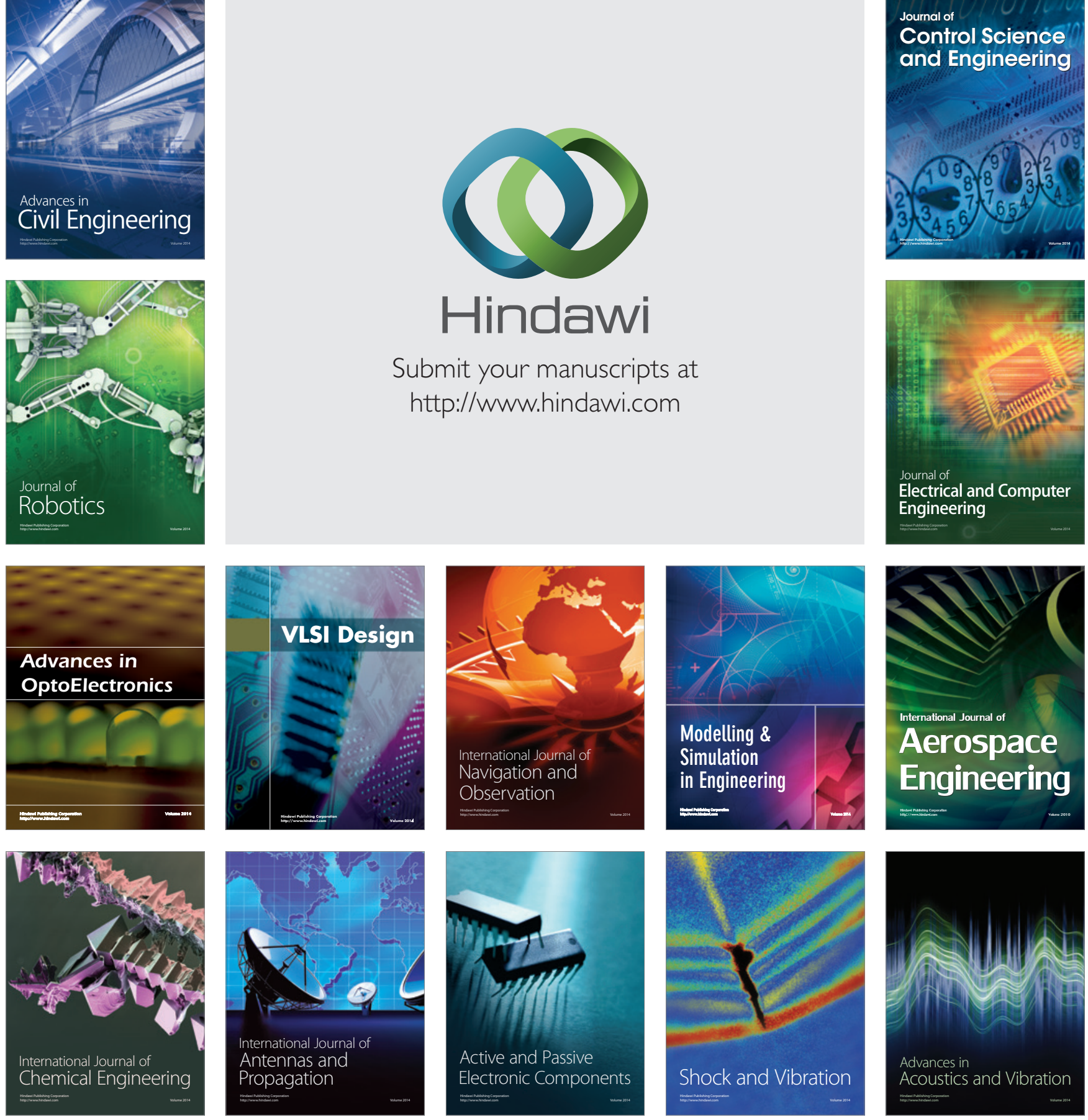